\title{
Giant outburst from the supergiant fast X-ray transient IGR J17544-2619: accretion from a transient disc?^
}

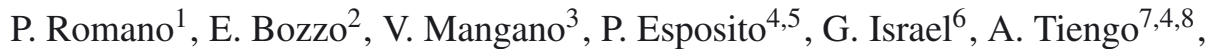 \\ S. Campana ${ }^{9}$, L. Ducci ${ }^{10,2}$, C. Ferrigno ${ }^{2}$, and J. A. Kennea ${ }^{3}$ \\ 1 INAF, Istituto di Astrofisica Spaziale e Fisica Cosmica - Palermo, via U. La Malfa 153, 90146 Palermo, Italy \\ e-mail: romano@ifc.inaf.it \\ 2 ISDC Data Center for Astrophysics, Université de Genève, 16 chemin d'Écogia, 1290 Versoix, Switzerland \\ 3 Department of Astronomy and Astrophysics, Pennsylvania State University, University Park, PA 16802, USA \\ 4 INAF, Istituto di Astrofisica Spaziale e Fisica Cosmica - Milano, via E. Bassini 15, 20133 Milano, Italy \\ 5 Harvard-Smithsonian Center for Astrophysics, 60 Garden Street, Cambridge, MA 02138, USA \\ 6 INAF, Osservatorio Astronomico di Roma, via Frascati 33, 00040 Monte Porzio Catone, Italy \\ Istituto Universitario di Studi Superiori, Piazza della Vittoria 15, 27100 Pavia, Italy \\ INFN, Sezione di Pavia, via A. Bassi 6, 27100 Pavia, Italy \\ INAF, Osservatorio Astronomico di Brera, via E. Bianchi 46, 23807 Merate, Italy \\ 10 Institut für Astronomie und Astrophysik, Eberhard Karls Universität, Sand 1, 72076 Tübingen, Germany \\ Received 26 January 2015 / Accepted 12 February 2015
}

\section{ABSTRACT}

\begin{abstract}
Supergiant fast X-ray transients (SFXTs) are high mass X-ray binaries associated with OB supergiant companions and characterized by an X-ray flaring behaviour whose dynamical range reaches 5 orders of magnitude on time scales of a few hundred to thousands of seconds. Current investigations concentrate on finding possible mechanisms to inhibit accretion in SFXTs and to explain their unusually low average X-ray luminosity. We present the Swift observations of an exceptionally bright outburst displayed by the SFXT IGR J17544-2619 on 2014 October 10 when the source achieved a peak luminosity of $3 \times 10^{38} \mathrm{erg} \mathrm{s}^{-1}$. This extends the total source dynamic range to $\gtrsim 10^{6}$, the largest (by a factor of 10) recorded so far from an SFXT. Tentative evidence for pulsations at a period of $11.6 \mathrm{~s}$ is also reported. We show that these observations challenge, for the first time, the maximum theoretical luminosity achievable by an SFXT and propose that this giant outburst was due to the formation of a transient accretion disc around the compact object.
\end{abstract}

Key words. X-rays: binaries - X-rays: individuals: IGR J17544-2619 - accretion, accretion disks

\section{Introduction}

Supergiant fast X-ray transients (SFXTs) are high mass X-ray binaries (HMXBs) hosting a neutron star (NS) and an OB supergiant companion (Sguera et al. 2005; Negueruela et al. 2006) which display X-ray flares reaching, for a few hours, $10^{36}-10^{37} \mathrm{erg} \mathrm{s}^{-1}$ (see Romano et al. 2014c, for a recent review). This is at odds with normal supergiant HMXBs, which display a fairly constant average luminosity with typical variations of a factor of 10-50 on time scales of a few hundred to thousands of seconds; SFXTs are also significantly subluminous with respect to classical Sg-HMXBs like Vela X-1 (Bozzo et al. 2015), and show a dynamical range up to 5 orders of magnitude, as their luminosities can be as low as $\sim 10^{32} \mathrm{erg} \mathrm{s}^{-1}$ during quiescence (e.g. in 't Zand 2005; Bozzo et al. 2010).

The transient IGR J17544-2619 (Sunyaev et al. 2003) is one of the SFXT prototypes in which a NS orbits an O9Ib star located at $3.6 \mathrm{kpc}$ (Pellizza et al. 2006; Rahoui et al. 2008) every $4.926 \pm 0.001 \mathrm{~d}$ (Clark et al. 2009). It has been showing large flux swings since its discovery (in't Zand et al. 2004; in 't Zand 2005; Rampy et al. 2009; Romano et al. 2014c), with the brightest flux recorded by Swift/XRT (Burrows et al. 2005) during the 2013 June 28 outburst (Romano et al. 2013a) at $\sim 6.5 \times 10^{-9} \mathrm{erg} \mathrm{cm}^{-2} \mathrm{~s}^{-1}(0.3-10 \mathrm{keV}$, unabsorbed)

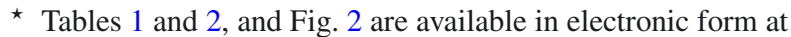
http://www. aanda.org corresponding to $L \sim 10^{37} \mathrm{erg} \mathrm{s}^{-1}$. These properties were initially interpreted in terms of accretion onto the NS from what in 't Zand (2005) calls a "clumpy wind". However, as discussed in Bozzo et al. (2013), we now know that the X-ray variability of SFXTs cannot be easily reconciled with a simple extreme clumpy wind model. Alternatives have been proposed to explain the large X-ray luminosity swings, invoking either the presence of magnetic/centrifugal barriers (Grebenev \& Sunyaev 2007; Bozzo et al. 2008) or a subsonic settling accretion regime (Shakura et al. 2014). A recent NuSTAR observation (Bhalerao et al. 2015), revealed a cyclotron line at $17 \mathrm{keV}$ in IGR J17544-2619, yielding the first measurement of the magnetic field in an SFXT at $\sim 1.5 \times 10^{12} \mathrm{G}$ as typical of accreting NS in HMXBs.

In this Letter we present the extraordinary set of observations collected by Swift during the 2014 October 10 outburst of IGR J17544-2619 (Sect. 2), during which the source reached an unabsorbed mean flux of $\sim 4.8 \times 10^{-8} \mathrm{erg} \mathrm{cm}^{-2} \mathrm{~s}^{-1}(0.3-10 \mathrm{keV}$; Sect. 3.1) thus exceeding all preceding records by a factor of 10. During this event evidence for a transient pulsation at $\sim 12 \mathrm{~s}$ (Sect. 3.2) and an expanding X-ray halo around the source were found in the XRT data.

\section{Observations and data reduction}

IGR J17544-2619 triggered Swift/BAT (Barthelmy et al. $2005)$ on 2014 October 10 at $T_{0}=15: 04: 19$ UT 

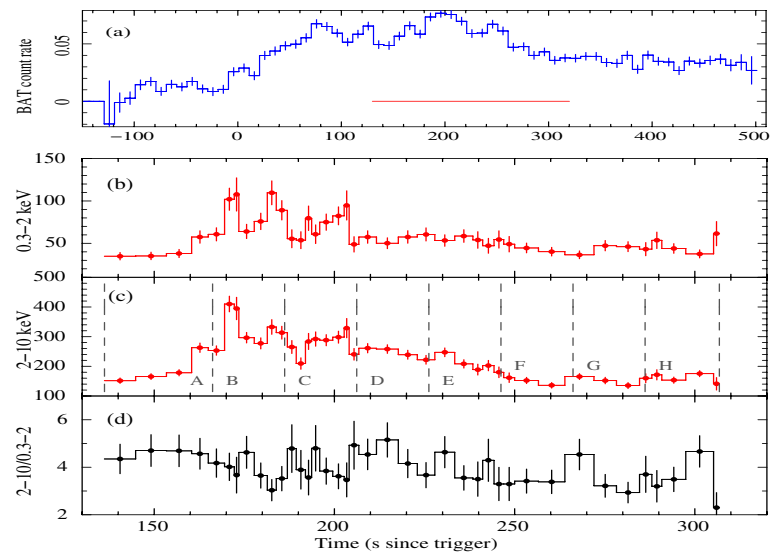

Fig. 1. Light curves of the 2014 October 10 outburst of IGR J17544 -2619. a) BAT $(14-150 \mathrm{keV}, 10 \mathrm{~s}$ binning): the red horizontal line marks the XRT data span; b) XRT soft band $(0.3-2 \mathrm{keV}$, at 30 counts bin $\left.{ }^{-1}\right)$; c) XRT hard band $\left(2-10 \mathrm{keV}\right.$, at 30 counts bin $\left.{ }^{-1}\right)$; d) XRT hardness ratio $(2-10 \mathrm{keV} / 0.3-2 \mathrm{keV})$. The dashed vertical lines mark the time selections for BAT and XRT spectroscopy (Sect. 3.1).

(image trigger $=614903$, Romano et al. 2014b). Swift immediately slewed to the target and the narrow-field instruments started observing at $T_{0}+132 \mathrm{~s}$. The automated target (AT, sequence 00614903000) ran for four orbits, until $\left.T_{0}+17.5 \mathrm{ks}\right)$. Follow-up target of opportunity observations were obtained (sequences 00035056161-166). The data cover the first $5 \mathrm{~d}$ after the beginning of the outburst, with six Swift observations for an XRT exposure of $\sim 12.5 \mathrm{ks}$ (see Table 1). Based on the most recent outburst ephemeris for this source $(P=4.92693 \pm$ $0.00036 \mathrm{~d}$, periastron at MJD $53732.65 \pm 0.23$; Smith 2014), the outburst started at phase $\phi=0.11$.

The data were processed and analysed using the standard software (FTOOLS v6.16), calibration (CALDB 20140709), and methods. Background-subtracted BAT light curves were created in the standard energy bands and mask-weighted spectra were extracted during the first orbit of data. The XRT data were processed and filtered with XRTPIPELINE (v0.13.1). Pileup was corrected for (ObsID 00614903000 only) by adopting standard procedures (Romano et al. 2006; Vaughan et al. 2006): source events were extracted from annuli (inner/outer radii of 4/20 pix for windowed-timing (WT), 5/30 pix for photon-counting (PC); 1 pixel $\left.\sim 2.36^{\prime \prime}\right)$. Light curves were corrected for point spread function losses, vignetting and were background subtracted. For our spectral analysis, we extracted events in the same regions as those adopted for the light curve creation and only considered grade 0 events for WT data. For our timing analysis, performed on the grade $0-2$ WT events, we also converted the event arrival times to the solar system barycentre with BARYCORR. Archival XRT data were also considered and similarly processed, from the following bright outbursts: 2011 March 24 (Farinelli et al. 2012, $\phi=0.96$ ), 2013 June 28 (Romano et al. 2013a, $\phi=$ 0.86); 2013 September 11 (Romano et al. 2013b, $\phi=0.15$ ); 2014 May 25 (Romano et al. 2014a, $\phi=0.16$ ).

\section{Analysis and results}

\subsection{A giant outburst?}

Figure 1 shows the Swift BAT and XRT light curves of the first orbit of data (before the first Earth occultation). While no spectral variations could be detected in the BAT data, the XRT data show variations in the hardness ratio. To investigate their nature, we extracted simultaneous XRT and BAT spectra in eight time

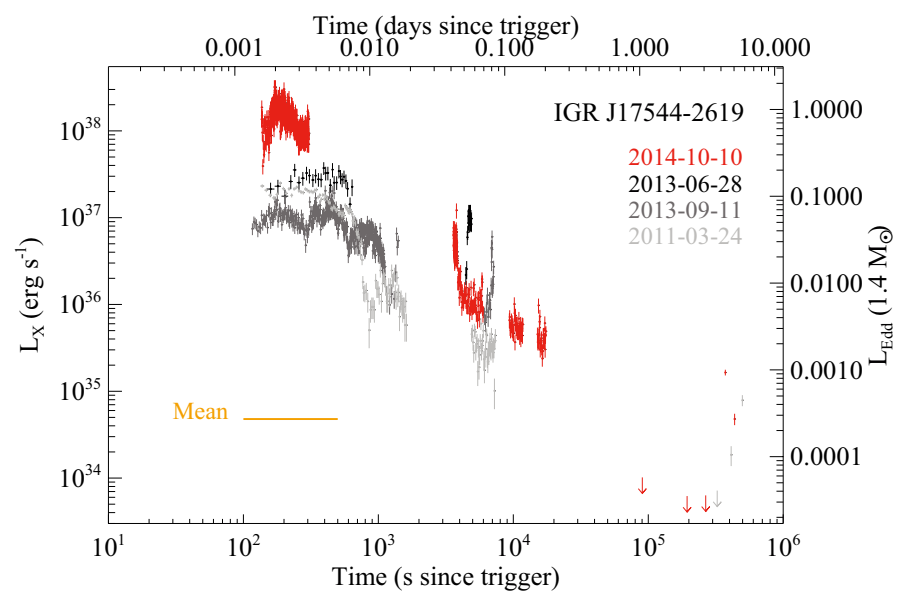

Fig. 3. Bolometric X-ray luminosity light curves of the brightest outbursts recorded by Swift for this source. Red: 2014 October 10, light grey: 2011 March 24, black: 2013 June 28, dark grey: 2013 September 11. The horizontal (orange) line marks the average level for this source.

intervals (marked A to H in Fig. 1c), as well as in the total (Orb 1, see Fig. 2), and fit them in the $0.3-10 \mathrm{keV}$ and $14-70 \mathrm{keV}$ bands, respectively (see Table 2), with an absorbed power law with a high energy cut-off (PHABS*POWER *HIGHECUT in XSPEC). Even this simple spectral model does not allow us to firmly establish whether variations of the absorption or intrinsic emission have a leading role in the hardness ratio modulation.

The 2014 October 10 outburst exceeded all preceding records. The XRT light curve reached 668 counts $^{-1}$ when binned at a signal-to-noise ratio $S / N=5$, which converts to a peak flux of $1.0 \times 10^{-7} \mathrm{erg} \mathrm{cm}^{-2} \mathrm{~s}^{-1}(0.3-10 \mathrm{keV}$, unabsorbed, $2.1 \mathrm{Crab})$, or $L \sim 3 \times 10^{38} \mathrm{erg} \mathrm{s}^{-1}$, when considering a count rate to flux conversion factor $\left(1.5 \times 10^{-10} \mathrm{erg} \mathrm{cm}^{-2}\right.$ count $\left.^{-1}\right)$ derived from the strictly simultaneous XRT+BAT spectra during interval B (Table 2).

Figure 3 shows the bolometric X-ray luminosity light curve of the 2014 October 10 outburst compared to the brightest ones recorded by Swift for this source. The right-hand $y$-axis is the standard Eddington luminosity for spherical accretion of fully ionized hydrogen for a $1.4 M_{\odot}$ NS according to $L_{\text {Edd }} \simeq$ $1.26 \times 10^{38}\left(M / M_{\odot}\right) \operatorname{erg~s}^{-1}$ (Basko \& Sunyaev 1975, we neglected any effect on the Eddington luminosity due to the high NS magnetic field as this is not relevant for our conclusions, see Sect. 4). We also mark the out-of-outburst average count rate obtained during the two-year monitoring with Swift/XRT (Romano et al. 2011), 0.1 counts $\mathrm{s}^{-1}$, implying an average flux of $\sim 1.5 \times 10^{-11} \mathrm{erg} \mathrm{cm}^{-2} \mathrm{~s}^{-1}(0.3-10 \mathrm{keV}$, unabsorbed) and bolometric luminosity of $\sim 4.7 \times 10^{34} \mathrm{erg} \mathrm{s}^{-1}$.

\subsection{Pulsations}

For the timing analysis, we concentrated on the WT mode (time resolution: $1.78 \mathrm{~ms}$ ) XRT data of the first orbit, since this segment is an uninterrupted stretch of data and contains most of the counts (more than $50 \%$ of the total). In the Fourier transform of the data, a peak $(v / \Delta v \sim 3)$ arises around $0.086 \mathrm{~Hz}$ ( $\sim 12 \mathrm{~s}$; see Fig. 4 , left). The period of this candidate modulation, as measured by a $Z^{2}$ test, is $11.58 \pm 0.03 \mathrm{~s}$. We estimated for this timing feature a quality factor (van der Klis 2000) of $Q=v / \Delta v \sim 3$. Although this would be comparable to the typical quality factors of quasi-periodic oscillations (QPOs; see e.g. van der Klis 2006, and references therein), the low Fourier resolution for frequencies around $0.1 \mathrm{~Hz}$ (see Fig. 4, left) would 

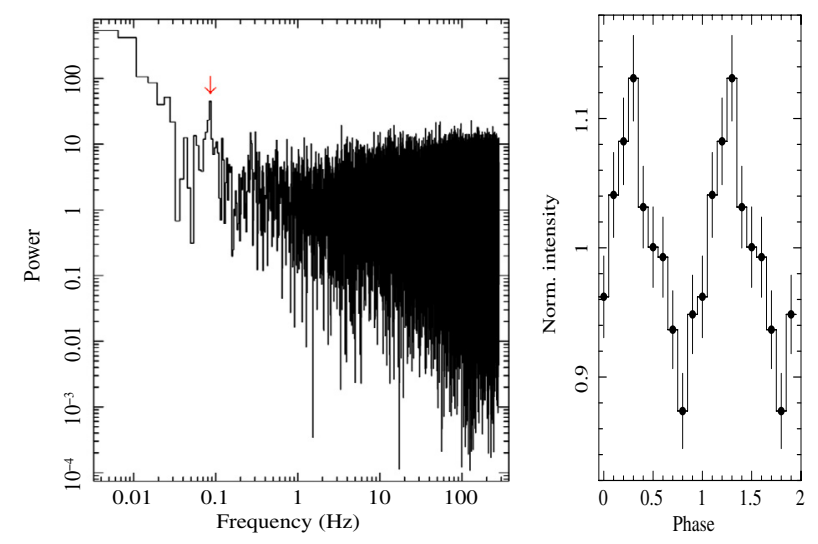

Fig. 4. Left: Fourier power spectrum of the Swift/XRT WT mode data $(0.3-10 \mathrm{keV})$ collected at the beginning of the outburst. The peak corresponding to the $0.086 \mathrm{~Hz}$ signal is indicated by the red arrow. Right: Folded profile obtained by folding the WT at the $11.6 \mathrm{~s}$ candidate periodicity (see Sect. 3.2 for details).

not allow us to distinguish the case of a QPO from that of a coherent pulsation. Given the apparent modulation of the source light curve at a period similar to the peak of the timing feature, we tentatively ascribe the latter to the pulsation of the NS hosted in IGR J17544-2619. The corresponding pulse profile is sinusoidal (Fig. 4, right), with an rms pulsed fraction of $9.1 \pm 0.6 \%$ (or $10 \pm 1 \%$ if the pulsed fraction is derived using a sinusoidal fit). This feature cannot be tracked down in the following WT or PC data and is embedded in a noisy spectrum, making it difficult to evaluate its significance.

We carefully inspected the light curve to check whether the $\sim 12 \mathrm{~s}$ signal is due to a modulation present along the whole dataset or, given the relatively short exposure, to the occurrence of one or a few burst-like events. As shown in Fig. 5, the light curve is characterized by strong aperiodic variability (which is the source of the red noise in the Fourier power spectrum) and by a number of superimposed peaks and dips, which are almost equidistant from each other, separated by about 11-12 s (the origin of the signal). These features are present during most, but not all of, the first orbit data. To evaluate the significance of the $\sim 12$ s modulation, we fit the light curve with a model consisting of a constant plus two Gaussians for the continuum (centred at about 8 and $40 \mathrm{~s}$; the one at $\sim 8 \mathrm{~s}$ with a negative normalization to account for the large dip around $5 \mathrm{~s}$ ), and added a sinusoidal component to account for the modulation. All parameters were left free to vary, and we obtained a value of $P=11.60 \pm 0.13 \mathrm{~s}$ $(1 \sigma)$ for the modulation period. We applied the F-test to assess whether the additional sinusoidal component needed to be included. We found that the probability of the inclusion being significant is of $99.984 \%$, corresponding to a detection at about $4 \sigma$ confidence level single-trial (period).

We also searched for pulsations in the data of the previous bright outbursts that afforded long observations and good counting statistics (see Sect. 2), but no significant signal was found at around $0.086 \mathrm{~Hz}$ or at different frequencies. In all cases we obtained $3 \sigma$ upper limits on the source pulse fraction of $\sim 10 \%$ for a coherent signal in the range of $0.005-20 \mathrm{~s}$.

\section{Discussion and conclusions}

Over the past few years SFXTs, and IGR J17544-2619 in particular with its uniquely high dynamic range now reaching $\gtrsim 10^{6}$, have been challenging our understanding of wind accretion onto NSs. In all the models proposed so far to interpret the peculiar behaviour of these sources in X-rays, a particular effort

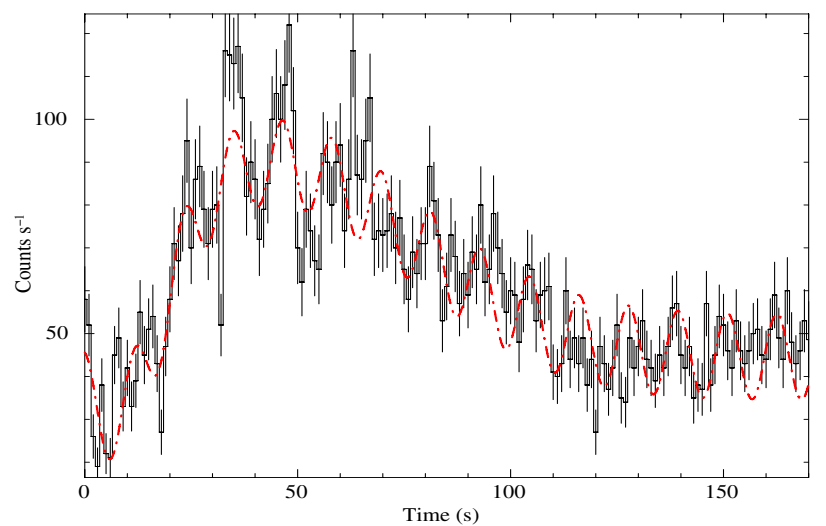

Fig. 5. Swift/XRT WT (0.3-10 keV) light curve (black) fitted with a model consisting of a constant plus two Gaussian for the continuum, and an additional sinusoidal component to account for the modulation.

was dedicated to finding a mechanism to inhibit accretion and provide a reliable explanation for their unusually low averaged $\mathrm{X}$-ray luminosity compared to classical systems. While different inhibition mechanisms could be considered to explain luminosities as low as $\sim 10^{32} \mathrm{erg} \mathrm{s}^{-1}$, in all cases it has been assumed that the maximum achievable luminosity is that corresponding to what is known as the direct-accretion regime, where all inhibiting mechanisms are overcome and the Bondi-Hoyle accretion rate sets in (see e.g. Shakura et al. 2012, for a recent review). As we show below, the peak X-ray luminosity of IGR J17544-2619 during the exceptional outburst reported in this paper reached the standard Eddington limit expected for a NS of $1.4 M_{\odot}$ and challenged for the first time the maximum (rather than the minimum) theoretical luminosity expected for an SFXT.

In the classical model of wind accretion, the maximum NS accretion rate is regulated by the accretion radius $R_{\mathrm{acc}} \simeq$ $2 G M_{\mathrm{NS}} / v_{\text {rel }}^{2}$, where $M_{\mathrm{NS}}$ is the NS mass, and $v_{\text {rel }}^{2}=v_{\text {orb }}(a)^{2}+$ $v_{\mathrm{w}}(a, t)^{2}$. In the latter expression, $v_{\text {orb }}(a)$ is the NS orbital velocity at a separation $a$ from its companion and $v_{\mathrm{w}}(a, t)$ is the companion wind velocity at the NS location and time $t$. The rate at which the wind material is captured and accreted by the NS is thus given by $\dot{M}_{\text {acc }}=\pi R_{\text {acc }}^{2} v_{\text {rel }} \rho_{\mathrm{w}}(a, t)$ (where $\rho$ is the wind density), and the correspondingly released X-ray luminosity can be estimated as $L_{\mathrm{X}}=G M \dot{M}_{\mathrm{acc}} / R_{\mathrm{NS}}$. Assuming the simplest case of a spherically symmetric wind and neglecting the NS orbital velocity, the wind density can be expressed at any time by $\rho_{\mathrm{w}}(a)=\dot{M}_{\mathrm{w}} /\left(4 \pi a^{2} v_{\mathrm{w}}(a)\right)$, where $\dot{M}_{\mathrm{w}}$ is the wind mass loss rate. The Bondi-Hoyle mass accretion rate can be expressed as

$L_{\mathrm{X}} \approx 5.8 \times 10^{35} \dot{M}_{-6} a_{5 \mathrm{~d}}^{-2} v_{8}^{-4} \mathrm{erg} \mathrm{s}^{-1}$,

where $\dot{M}_{-6}=\dot{M}_{\mathrm{w}} /\left(10^{-6} M_{\odot} \mathrm{yr}^{-1}\right), v_{8}=v_{\mathrm{w}} /\left(10^{8} \mathrm{~cm} \mathrm{~s}^{-1}\right)$. In Eq. (1) we assumed a circular orbit to express the separation $a$ as a function of the total mass $\left(M_{\mathrm{tot}}\right)$ and the orbital period $\left(P_{\mathrm{orb}}\right)$ of the system $a_{5 \mathrm{~d}}=2.7 \times 10^{12} P_{5 \mathrm{~d}}^{2 / 3} M_{30}^{1 / 3}$, where $P_{5 \mathrm{~d}}$ is the orbital period in units of $5 \mathrm{~d}$ and $M_{30}$ is the total mass of the system in units of $30 M_{\odot}$, chosen to qualitatively match the characteristic values of IGR J17544-2619 (see Sect. 1).

Equation (1) shows that the peak luminosity achieved by IGR J17544-2619 during the 2014 October 10 outburst can only be explained by assuming a very low wind velocity $\left(v_{\mathrm{w}} \lesssim\right.$ $\left.2 \times 10^{6} \mathrm{~cm} \mathrm{~s}^{-1}\right)$ or an unlikely high mass loss rate from the $\operatorname{star}^{1}\left(\dot{M}_{\mathrm{w}} \gg 10^{-6} M_{\odot} \mathrm{yr}^{-1}\right)$. Considering the first hypothesis,

1 The typical velocity of the wind from an OB supergiant can be as high as $1-2 \times 10^{8} \mathrm{~cm} \mathrm{~s}^{-1}$, and the mass loss rate is usually within the range $10^{-7}-10^{-5} M_{\odot} \mathrm{yr}^{-1}$ (see e.g. Puls et al. 2008, for a recent review). 
it is known that the photoionization of the wind material by the X-rays emitted from the NS can lead to a substantial reduction of the stellar wind velocity, especially in bright HMXBs endowed with short orbital periods and eccentric orbits (see e.g. Watanabe et al. 2006; Ducci et al. 2010, and references therein). The orbital period of IGR J17544-2619 could be sufficiently short and the orbit eccentric enough (Nikolaeva et al. 2013) to allow a substantial ionization of the wind material and lead to wind velocities as low as the values indicated above (at least during the brightest outbursts when the X-ray emission is more intense). If this is the case, it is difficult to avoid the formation of a temporary accretion disc around the NS in IGR J17544-2619, as a disc is already expected to be in place for wind velocities $\$ 5 \times 10^{7} \mathrm{~cm} \mathrm{~s}^{-1}$ (see discussion in Ducci et al. 2010). Fryxell et al. (1988) suggested that instabilities of inhomogeneous slow winds in HMXBs could lead not only to the rapid formation of accretion discs, but also to their quick dissipation (e.g. EXO 2030+375, Taam et al. 1988). On the one hand, accretion from a disc could already provide larger mass accretion rates than those typical of wind-fed binaries. On the other hand, their dissipation was shown to produce sudden increases in the mass accretion rates and the appearance of bright X-ray flares (by a factor of $\sim 10$ ). The typical duration of these flares is $\sim 6 G M_{\mathrm{NS}} / v_{\text {rel }}^{3}$, thus compatible with the observational properties of the super-outburst from IGR J17544-2619 (assuming the values mentioned above for $v_{\text {rel }}$ ). Additional simulations presented by Taam \& Fryxell (1988) also showed that a more erratic or periodic behaviour of the flares can be obtained by assuming different properties of the poorly known density and velocity asymmetry of the accretion flow. The presence of short-lived accretion discs in SFXTs (and other NS HMXBs in general) could be tested observationally through the detection of rapid spin-up phases (Klochkov et al. 2011; Jenke et al. 2012). However, IGR J17544-2619 has not displayed convincing evidence of pulsations in the past (see Sect. 1) and we could only reveal a marginally significant pulsed signal at $\sim 11.6 \mathrm{~s}$ during the peak of the event reported in this paper. No meaningful searches for spin period derivatives can thus be carried out.

We cannot currently rule out alternative explanations for the exceptionally bright outburst of IGR J17544-2619 recorded by Swift. A viable mechanism to overcome the difficulties in the standard Bondi-Hoyle accretion scenario is that of the "ingestion of a massive clump" (Bozzo et al. 2011). In this case, it is assumed that the bright outburst was due to a dense structure in the wind of the supergiant star being accreted onto the compact object. The equation relating the density $\rho_{\mathrm{w}}(a, t)$ and the mass loss rate from the supergiant introduced above is no longer valid, as massive structures are created by definition in asymmetric winds. A sufficiently high increase in the density of the accreting material (together with a possible decrease in the corresponding velocity) could thus be invoked to achieve sufficiently high $\dot{M}_{\text {acc }}$ without implying an enhanced $\dot{M}_{\mathrm{w}}$. Even though current multi-dimensional simulations of winds from massive stars do not favour the formation of large and very dense clumps (see the discussion in Bozzo et al. 2015, and references therein), at least in the case of IGR J18410-0535 X-ray observations have revealed some evidence for the ingestion of a dense structure onto the NS (Bozzo et al. 2011). The spectroscopic analysis of the outburst from IGR J17544-2619 did not reveal any change in the source spectral properties that could favour such a hypothesis. While it could still be assumed that this is due to projection effects between the velocity vector of the clump and the observed line of sight, in the present case we consider this explanation unlikely.

The detection of other bright outbursts from the SFXTs with Swift and, in the future, with the next generation of X-ray facilities providing fine X-ray spectroscopy on still unexplored short time scales (e.g. the LOFT/LAD; Feroci et al. 2014; Orlandini et al. 2015), will help clarify the origin of these events.

Acknowledgements. We wholeheartedly thank the Swift team duty scientists and science planners for their courteous efficiency, and S. D. Barthelmy, D. N. Burrows, and N. Gehrels. We thank S. E. Motta, L. Stella, A. Beardmore, M. Capalbi, and S. Vercellone for useful discussions. We also thank our referee for swift comments that helped improve the paper. P.R. and S.C. acknowledge contract ASI-INAF I/004/11/0. P.E. acknowledges a Fulbright Research Scholar grant administered by the US-Italy Fulbright Commission and is grateful to the Harvard-Smithsonian Center for Astrophysics for hosting him during his Fulbright exchange. L.D. thanks Deutsches Zentrum für Luft und Raumfahrt (Grant FKZ 50 OG 1301).

\section{References}

Barthelmy, S. D., Barbier, L. M., Cummings, J. R., et al. 2005, Space Sci. Rev., 120,143

Basko, M. M., \& Sunyaev, R. A. 1975, A\&A, 42, 311

Bhalerao, V., Romano, P., Tomsick, J., et al. 2015, MNRAS, 447, 2274

Bozzo, E., Falanga, M., \& Stella, L. 2008, ApJ, 683, 1031

Bozzo, E., Stella, L., Ferrigno, C., et al. 2010, A\&A, 519, A6

Bozzo, E., Giunta, A., Cusumano, G., et al. 2011, A\&A, 531, A130

Bozzo, E., Romano, P., Ferrigno, C., et al. 2013, Adv. Space Res., 51, 1593

Bozzo, E., Romano, P., Ducci, L., et al. 2015, Adv. Space Res., 55, 1255

Burrows, D. N., Hill, J. E., Nousek, J. A., et al. 2005, Space Sci. Rev., 120 165

Clark, D. J., Hill, A. B., Bird, A. J., et al. 2009, MNRAS, 399, L113

Drave, S. P., Bird, A. J., Sidoli, L., et al. 2014, MNRAS, 439, 2175

Ducci, L., Sidoli, L., \& Paizis, A. 2010, MNRAS, 408, 1540

Farinelli, R., Romano, P., Mangano, V., et al. 2012, MNRAS, 424, 2854

Feroci, M., den Herder, J. W., Bozzo, E., et al. 2014, SPIE Conf. Ser., 9144, 2

Fryxell, B. A., \& Taam, R. E. 1988, ApJ, 335, 862

Grebenev, S. A., \& Sunyaev, R. A. 2007, Astron. Lett., 33, 149

in 't Zand, J. J. M. 2005, A\&A, 441, L1

in 't Zand, J., Heise, J., Ubertini, P., Bazzano, A., \& Markwardt, C. 2004, ESA SP, 552, 427

Jenke, P., Finger, M. H., Wilson-Hodge, C. A., \& Camero-Arranz, A., 2012, ApJ, 759,124

Klochkov, D., Ferrigno, C., Santangelo, A., et al. 2011, A\&A, 536, L8 Negueruela, I., Smith, D. M., Harrison, T. E., \& Torrejón, J. M. 2006, ApJ, 638, 982

Nikolaeva, E. A., Bikmaev, I. F., Melnikov, S. S., et al. 2013, BCAO, 109, 27 Orlandini, M., Doroshenko, V., Zampieri, L., et al. 2015 [arXiv: 1501. 02777] Pellizza, L. J., Chaty, S., \& Negueruela, I. 2006, A\&A, 455, 653

Puls, J., Vink, J. S., \& Najarro, F. 2008, A\&ARv, 16, 209

Rahoui, F., Chaty, S., Lagage, P.-O., \& Pantin, E. 2008, A\&A, 484, 801

Rampy, R. A., Smith, D. M., \& Negueruela, I. 2009, ApJ, 707, 243

Romano, P., Campana, S., Chincarini, G., et al. 2006, A\&A, 456, 917

Romano, P., La Parola, V., Vercellone, S., et al. 2011, MNRAS, 410, 1825

Romano, P., Evans, P. A., Kennea, J. A., et al. 2013a, ATel, 5179, 1

Romano, P., Markwardt, C. B., Chester, M. M., et al. 2013b, ATel, 5388, 1

Romano, P., Barthelmy, S. D., Lien, A. Y., et al. 2014a, ATel, 6173, 1

Romano, P., Barthelmy, S. D., Mangano, V., et al. 2014b, ATel, 6566, 1

Romano, P., Krimm, H. A., Palmer, D. M., et al. 2014c, A\&A, 562, A2

Sguera, V., Barlow, E. J., Bird, A. J., et al. 2005, A\&A, 444, 221

Shakura, N., Postnov, K., Kochetkova, A., et al. 2012, MNRAS, 420, 216

Shakura, N., Postnov, K., Sidoli, L., \& Paizis, A. 2014, MNRAS, 442, 2325

Smith, D. M. 2014, ATel, 6227, 1

Stella, L., White, N. E., \& Rosner, R. 1986, ApJ, 308, 669

Sunyaev, R. A., Grebenev, S. A., Lutovinov, A. A., et al. 2003, ATel, 190, 1

Taam, R. E., \& Fryxell, B. A. 1988, ApJ, 327, L73

Taam, R. E., Brown, D. A., \& Fryxell, B. A. 1988, ApJ, 331, L117

van der Klis, M. 2000, ARA\&A, 38, 717

van der Klis, M. 2006, Adv. Space Sci., 38, 2675

Vaughan, S., Goad, M. R., Beardmore, A. P., et al. 2006, ApJ, 638, 920

Watanabe, S., Sako, M., Ishida, M., et al. 2006, ApJ, 651, 421 
Table 1. Observation log.

\begin{tabular}{|c|c|c|c|c|}
\hline Sequence & Obs/Mode & $\begin{array}{l}\text { Start time } \\
\text { (UT) }\end{array}$ & $\begin{array}{l}\text { End time (UT) } \\
\text { (UT) }\end{array}$ & $\begin{array}{r}\text { Expo. } \\
(s)\end{array}$ \\
\hline 00614903000 & BAT/evt & 2014-10-10 15:00:30 & 2014-10-10 20:00:06 & 1648 \\
\hline 00614903000 & XRT/WT & & 2014-10-10 19:15:26 & 420 \\
\hline 00614903000 & XRT/PC & 2014-10-10 16:08:27 & 2014-10-10 19:56:22 & 7173 \\
\hline 00035056161 & XRT/PC & 2014-10-11 16:02:14 & 2014-10-11 16:18:45 & 973 \\
\hline 00035056162 & $\mathrm{XRT} / \mathrm{PC}$ & $-1220: 49: 24$ & $2014-10-12$ 21:05:51 & 978 \\
\hline 00035056163 & $\mathrm{XRT} / \mathrm{PC}$ & 2014-10-13 16:29:14 & 2014-10-13 17:40:37 & 960 \\
\hline 00035056165 & XRT/PC & 2014-10-14 22:26:08 & 2014-10-14 22:41:54 & 925 \\
\hline 00035056166 & $\mathrm{XRT} / \mathrm{PC}$ & 2014-10-15 1 & 2014-10-15 & 1071 \\
\hline $00449907000^{a}$ & XRT/WT & 2011-03-24 01:59:15 & 2011-03-24 03:18:22 & 657 \\
\hline & & & & 3360 \\
\hline $00035056150^{a}$ & XRT/PC & $2011-03-27$ 19:54:23 & $2011-03-2720: 11: 56$ & 1046 \\
\hline $00035056151^{a}$ & XRT/PC & & & 662 \\
\hline $00035056152^{a}$ & $\mathrm{XRT} / \mathrm{PC}$ & $-2920: 15: 13$ & 2011-0 & 504 \\
\hline 00559221000 & XRT/PC & $6-28.07 \cdot 28 \cdot 56$ & $2808: 48: 24$ & 1010 \\
\hline & & & & 1024 \\
\hline 00570402000 & XRT/PC & 2013-09-11 16:18:49 & 2013-09-11 17:59:42 & 1394 \\
\hline 00599954000 & XRT/WT & $2014-05-25$ 22:33:22 & 2014-05-25 22:34:31 & \\
\hline 00599955000 & XRT/WT & $2014-05-25$ 22:34:54 & $2014-05-25$ 22:55:55 & 1235 \\
\hline 00035056156 & XRT/PC & $2014-05-26$ 19:03:35 & $2014-05-26$ 19:20:05 & 978 \\
\hline 00035056157 & XRT/PC & & $2014-05-27$ 16:30:56 & 920 \\
\hline 00035056158 & XRT/PC & 2014-05-30 16:16:27 & 2014-05-30 22:38:53 & 1241 \\
\hline 00035056159 & XRT/PC & $2014-05-31$ 19:11:56 & 2014-05-31 21:00:54 & 1168 \\
\hline 00035056160 & XRT/PC & 2014-06-01 09:27:33 & 2014-06-01 21:09:54 & 1133 \\
\hline
\end{tabular}

References. ${ }^{(a)}$ See Farinelli et al. (2012).

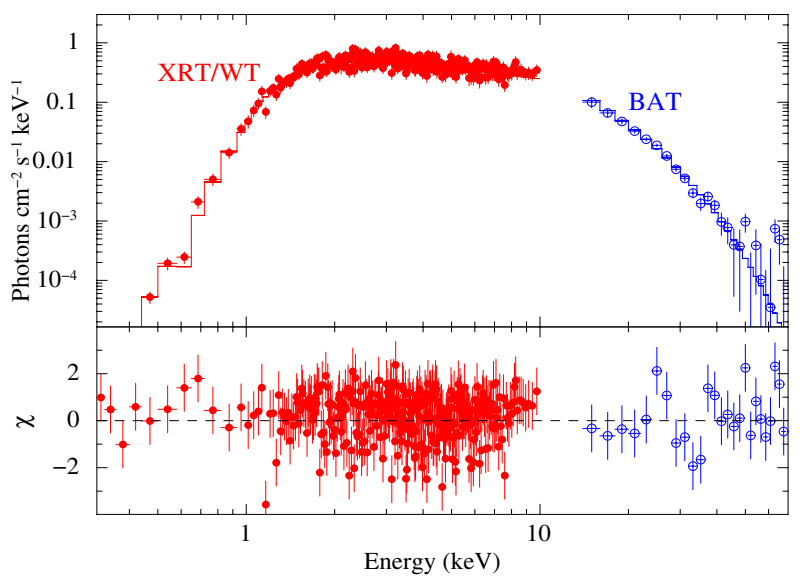

Fig. 2. Spectroscopy of the 2014 October 10 outburst of IGR J17544-2619. Top panel: simultaneous XRT/WT data (filled red circles) and BAT data (empty blue circles) fit with an absorbed power law with a high energy exponential cut-off model. Bottom panel: residuals of the fit (in units of standard deviations).

Table 2. Spectral fits of simultaneous XRT/WT and BAT data.

\begin{tabular}{|c|c|c|c|c|c|c|c|c|}
\hline Spectrum $^{a}$ & $\begin{array}{c}\text { Time } \\
\text { (s since } T_{0} \text { ) }\end{array}$ & $\begin{array}{c}N_{\mathrm{H}} \\
\left(\times 10^{22} \mathrm{~cm}^{-2}\right)\end{array}$ & $\Gamma$ & $\begin{array}{c}E_{\text {fold }} \\
(\mathrm{keV})\end{array}$ & $\begin{array}{c}E_{\mathrm{cut}} \\
(\mathrm{keV})\end{array}$ & $\begin{array}{c}\text { Flux }^{b} \\
\left(\times 10^{-8} \mathrm{erg} \mathrm{cm}^{-2} \mathrm{~s}^{-1}\right) \\
(0.3-10 \mathrm{keV}) \\
\end{array}$ & $\begin{array}{c}\text { Luminosity }^{c} \\
\left(\times 10^{37} \mathrm{erg} \mathrm{s}^{-1}\right) \\
(0.01-1000 \mathrm{keV}) \\
\end{array}$ & $\chi_{v}^{2} /$ d.o.f. \\
\hline Orb 1 & $136.2-306.8$ & $1.31_{-0.10}^{+0.11}$ & $0.72_{-0.07}^{+0.07}$ & $11.9_{-1.0}^{+1.0}$ & $6.7_{-0.4}^{+0.4}$ & $3.1_{-0.1}^{+0.1}$ & 10.0 & $1.01 / 364$ \\
\hline A & $136.2-166.2$ & $1.37_{-0.29}^{+0.34}$ & $0.70_{-0.20}^{+0.21}$ & $11.4_{-1.9}^{+2.0}$ & $6.6_{-0.7}^{+0.8}$ & $2.7_{-0.1}^{+0.1}$ & 8.8 & $0.92 / 83$ \\
\hline B & $166.2-186.2$ & $1.51_{-0.25}^{+0.29}$ & $0.85_{-0.17}^{+0.18}$ & $11.2_{-1.9}^{+1.9}$ & $6.7_{-0.7}^{+0.9}$ & $4.8_{-0.2}^{+0.1}$ & 15.3 & $0.84 / 99$ \\
\hline Peak $^{d}$ & $169.6-172.8$ & - & - & - & - & 10.0 & 32.0 & - \\
\hline $\mathrm{C}$ & 186.2-206.2 & $1.35_{-0.26}^{+0.29}$ & $0.71_{-0.19}^{+0.20}$ & $11.1_{-1.9}^{+1.9}$ & $6.7_{-0.7}^{+0.8}$ & $4.1_{-0.2}^{+0.2}$ & 13.2 & $1.10 / 89$ \\
\hline D & $206.2-226.2$ & $1.51_{-0.30}^{+0.35}$ & $0.86_{-0.21}^{+0.22}$ & $11.7_{-2.4}^{+2.5}$ & $7.6_{-1.0}^{+1.2}$ & $3.3_{-0.2}^{+0.2}$ & 10.7 & $1.06 / 77$ \\
\hline $\mathrm{E}$ & $226.2-246.2$ & $1.32_{-0.26}^{+0.30}$ & $0.77_{-0.19}^{+0.21}$ & $9.1_{-2.5}^{+2.4}$ & $7.9_{-0.9}^{+1.1}$ & $3.3_{-0.2}^{+0.2}$ & 10.6 & $0.98 / 72$ \\
\hline $\mathrm{F}$ & $246.2-266.2$ & $0.88_{-0.29}^{+0.20}$ & $0.48_{-0.22}^{+0.19}$ & $10.3_{-2.3}^{-2.5}$ & $6.7_{-0.8}^{-1.0}$ & $2.3_{-0.1}^{-0.2}$ & 7.3 & $0.98 / 56$ \\
\hline G & $266.2-286.2$ & $1.03_{-0.31}^{+0.37}$ & $0.51_{-0.23}^{+0.25}$ & $10.5_{-2.4}^{+2.2}$ & $6.2_{-0.8}^{+1.0}$ & $2.5_{-0.2}^{+0.2}$ & 8.0 & $0.89 / 58$ \\
\hline $\mathrm{H}$ & $286.2-306.8$ & $0.83_{-0.24}^{+0.30}$ & $0.55_{-0.20}^{+0.22}$ & $9.7_{-2.2}^{+2.1}$ & $6.0_{-0.9}^{+1.1}$ & $2.5_{-0.1}^{+0.1}$ & 8.0 & $0.99 / 62$ \\
\hline
\end{tabular}

Notes. ${ }^{(a)}$ Defined in Sect. 3.1, see Fig. 1c; ${ }^{(b)}$ average unabsorbed $0.3-10 \mathrm{keV}$ fluxes in units of $10^{-8}$ erg $\mathrm{cm}^{-2} \mathrm{~s}^{-1}$; ${ }^{(c)}$ bolometric luminosity in units of $10^{37} \mathrm{erg} \mathrm{s}^{-1}$; (d) the peak luminosity, calculated by converting peak count rate of 668 counts s${ }^{-1}$ by using the spectral parameters of spectrum B. 